\title{
Cycles in 5-connected triangulations
}

\author{
Alahmadi, A.; Aldred, R.E.L.; Thomassen, Carsten
}

Published in:

Journal of Combinatorial Theory. Series B

Link to article, DOI:

10.1016/j.jctb.2019.04.005

Publication date:

2020

Document Version

Peer reviewed version

Link back to DTU Orbit

Citation $(A P A)$ :

Alahmadi, A., Aldred, R. E. L., \& Thomassen, C. (2020). Cycles in 5-connected triangulations. Journal of Combinatorial Theory. Series B, 140, 27-44. https://doi.org/10.1016/j.jctb.2019.04.005

\section{General rights}

Copyright and moral rights for the publications made accessible in the public portal are retained by the authors and/or other copyright owners and it is a condition of accessing publications that users recognise and abide by the legal requirements associated with these rights.

- Users may download and print one copy of any publication from the public portal for the purpose of private study or research.

- You may not further distribute the material or use it for any profit-making activity or commercial gain

- You may freely distribute the URL identifying the publication in the public portal

If you believe that this document breaches copyright please contact us providing details, and we will remove access to the work immediately and investigate your claim. 


\title{
Journal of Combinatorial Theory, Series B

\section{Cycles in 5-connected triangulations}

\author{
A. Alahmadi ${ }^{a}$, R.E.L. Aldred ${ }^{\mathrm{b}}$, C. Thomassen ${ }^{\mathrm{c}}$ \\ a MECAA, Math Dept of King Abdulaziz University, Jeddah, Saudi Arabia \\ b Department of Mathematics and Statistics, University of Otago, P.O. Box 56, \\ Dunedin, New Zealand \\ c Department of Applied Mathematics and Computer Science, Technical University \\ of Denmark, DK-2800 Lyngby, Denmark
}

\section{A R T I C L E I N F O}

\section{Article history:}

Received 11 November 2015

Available online $\mathrm{xxxx}$

\section{Keywords:}

Cycles

Triangulation of a surface

Planar triangulation

Projective planar triangulation

\begin{abstract}
A B S T R A C T
We show that in 5-connected planar and projective planar triangulations on $n$ vertices, the number of Hamiltonian cycles grows exponentially with $n$. The result is best possible in the sense that 4-connected triangulations on $n$ vertices on any fixed surface may have only polynomially many cycles. Also, there is an infinite class of 5-connected graphs (not on a fixed surface) which have only polynomially many cycles. The result also extends to 5 -connected triangulations of the torus if a long standing conjecture of Nash-Williams holds. For any fixed surface, we show that every 5-connected triangulation of large face-width on that surface contains exponentially many cycles.
\end{abstract}

(C) 2019 Elsevier Inc. All rights reserved.

\section{Introduction}

Determining the number of cycles in a graph is a natural problem to consider in Graph Theory and has considerable application in, for example, Coding Theory (see [1-3]). We

E-mail addresses: adelnife2@yahoo.com (A. Alahmadi), raldred@maths.otago.ac.nz (R.E.L. Aldred), ctho@dtu.dk (C. Thomassen). 
are interested in determining the existence of exponentially many cycles in a broad class of sparse graphs. The quantity $f_{k}(n)$, defined to be the smallest number of cycles present in a cyclically $k$-edge-connected cubic graph on $n$ vertices, was introduced and investigated by Barefoot, Clark and Entringer [5] who proved that $f_{1}(n)=(3 n+26) / 4$ and $f_{2}(n)=\left(n^{2}+14 n\right) / 8$ for infinitely many $n$. Aldred and Thomassen [4] proved that $2^{n^{0.17}} \leq f_{3}(n) \leq 2^{n^{0.95}}$, indicating a marked difference between 2-connected and 3 -connected cubic graphs. While a subexponential bound was established for 3 -connected cubic graphs in general, it is quite possible that 3-connected cubic Hamiltonian graphs, for example, will always have exponentially many cycles. There are many other subclasses of 3-connected cubic graphs which may always have exponentially many cycles, including the cyclically-4-edge-connected cubic graphs. As observed in [5], for any graph to have a large number of cycles of any kind, it must have cycles which are long in relation to the number of vertices in the graph. It makes sense then to consider graphs which have Hamiltonian cycles.

The planar dual of a cyclically-5-edge-connected cubic planar graph is a 5-connected planar triangulation. Such graphs are well known to be Hamiltonian. Böhme, Harant and Tkáč [6] showed that in a 5-connected planar triangulation on $n$ vertices, there are at least $2^{O\left(n^{(1 / 4)}\right)}$ Hamiltonian cycles. They established a similar lower bound for projective planar triangulations of face-width at least 5 . We, too, consider the minimum number of Hamiltonian cycles that must be present in a 5-connected triangulation on the sphere, the projective plane, the torus or the Klein bottle and we consider the number of cycles in all surfaces. In all cases we obtain an exponential lower bound. With this in mind, it is worthwhile to note the following regarding any relaxation of our conditions on the class:

1. A 4-connected planar triangulation may have only polynomially many cycles as shown by the double wheel. (In [8] it was proved that any 4-connected planar triangulation has at least $n / \log (n)$ Hamiltonian cycles.)

2. More generally, a 4-connected Hamiltonian triangulation on any fixed surface may have at most $O\left(n^{4}\right)$ cycles. This can be seen by taking a fixed Hamiltonian triangulation on the surface and repeatedly subdividing an edge, making each new vertex adjacent to both vertices completing triangular faces on the original edge.

3. A 5-connected graph may have only polynomially many cycles as shown by a wheel with the centre vertex blown up to a triangle.

In Section 2 we will outline our approach and develop the preliminary results required to work on 5-connected planar triangulations. In Section 3 we apply the results and method to show that there are exponentially many Hamiltonian cycles in a 5-connected planar triangulation. We investigate extensions of the approach to surfaces other than the plane in Section 4. In the first subsection we note that our planar analysis can be repeated with minor modification if the face-width of our graphs is at least 7 . This gives us exponentially many Hamiltonian cycles in 5-connected triangulation of the projective 
plane (and the torus, if Nash-Williams' conjecture on the existence of a Hamiltonian cycle in every 4-connected toroidal graph [10] holds, and the Klein bottle if the obvious analogue of this conjecture also holds) with face-width at least 7 . We also note that a result of Böhme, Mohar and Thomassen [7] can be used in combination with our method to determine the existence of exponentially many cycles in suitably large face-width 5 -connected triangulations of any surface. In the second subsection, we develop a more general topological approach to enable us to apply our planar method to 5-connected triangulations of any surface. With this we are able to remove the face-width restriction for earlier results on the projective plane, torus and Klein bottle.

We use the terminology of [9] for all topological graph theory. In particular for definitions of triangulation, surface, face-width, genus and Euler genus. Also, our graphs have no loops or multiple edges unless explicitly stated otherwise.

\section{Preliminaries}

The key to our approach is the following remarkable result due to Tutte [13].

Theorem 1 ([13]). Let $G$ be a 4-connected planar graph. Then $G$ is Hamiltonian.

For a simple proof of this result see [12]. We shall also make use of the following well-known observation.

Lemma 1. If $G$ is a planar triangulation and $A$ is a minimal separating vertex set (that $i s$, the deletion of $A$ leaves a disconnected graph whereas the deletion of any proper subset of $A$ leaves a connected graph), then the subgraph of $G, G[A]$, induced by $A$ is a chordless cycle.

Proof. Let $G, A$ be as in the statement of the lemma and let $v$ be any vertex in $A$. As $A-v$ is not separating, $v$ has two neighbours $x, y$ belonging to distinct components of $G-A$. Hence the cycle $C$ in $G$ consisting of the neighbours of $v$ contains two vertices $v_{1}, v_{2}$ in $A$ such that $C-v_{1}-v_{2}$ has $x, y$ in distinct components. It follows that $v$ has degree at least 2 in $G[A]$. So $G[A]$ has a cycle $C^{\prime}$. Moreover the argument above shows that $C^{\prime}$ can be chosen so that every vertex on $C^{\prime}$ (except possibly one) has an incident edge going inside the cycle $C^{\prime}$ and one going outside $C^{\prime}$. Hence there must be at least one vertex inside (respectively outside) $C^{\prime}$, and hence $C^{\prime}$ is separating. As $A$ is minimal, $A=V\left(C^{\prime}\right)$, and $C^{\prime}$ is chordless.

Lemma 1 cannot be extended to higher surfaces as shown by easy examples on the torus. However, if $G$ is a triangulation of the torus (or any other fixed surface), and $A$ is a minimal separating vertex set of cardinality at most the length of a shortest non-contractible cycle, then a repetition of the proof of Lemma 1 shows that $G[A]$ is a chordless surface separating cycle. (A cycle $C$ is surface separating, if the surface contains 
two points not in $C$ which cannot be joined by a curve without intersecting $C$. For a more formal definition, see [9, page 104].) With a little more effort one can show that, if $A$ is a minimal separating vertex set with 4 vertices, then $G[A]$ is a surface separating cycle (but possibly with one or two chords).

In order to prove Theorem 2 below, we wish to find a positive real constant $c$ such that, for each 5-connected planar triangulation $G$, there is a subset $S \subseteq V(G)$ of independent vertices of degrees 5 or 6 , with $|S| \geq c|V(G)|$ and each subset $F \subset E(G)$ consisting of one edge incident with each vertex in $S$ has the property that $G-F$ is 4 -connected. Thus, by Theorem 1, G-F is Hamiltonian. By counting the number of Hamiltonian cycles we obtain, after factoring out double counting, the results we claim.

In the remainder of this section we will put together the results we need to determine a subset $S \subseteq V(G)$ with the desired properties.

First we focus on the distribution of vertices of degrees 5 and 6 with respect to 5 -cycles in a 5-connected planar triangulation $G$. As many of our arguments will refer to the embedding of our graph in the plane, our statements will be in terms of plane triangulations. Our first result makes use of colouring, and the following definition and terminology will facilitate the statement and proof of the result.

Definition. A graph $G$ is $k$-degenerate if each subgraph of $G$ contains a vertex of degree at most $k$.

It is well known that a $k$-degenerate graph has chromatic number at most $k+1$.

If $G$ is a plane graph, then each cycle $C$ in $G$ divides the plane into a bounded region and an unbounded region. We shall use interior of $C$ to refer to the bounded region bounded by $C$ together with the vertices and edges in $C$ itself. To refer to the interior of $C$ without the vertices and edges of $C$ being included, we use the term strict interior of $C$.

Lemma 2. Let $G$ be a 5-connected plane triangulation with $n$ vertices. Let $S$ be an independent set of vertices of degree 5 or 6 . Let $Q$ be the graph defined on $S$ such that two vertices are adjacent in $Q$ iff they lie on a common 5 -cycle in $G$. Then $Q$ is 12 -degenerate and therefore has chromatic number at most 13.

Proof. Let $G, S, Q$ be as in the statement of the lemma and let $x$ be any vertex in $S$. We say that a 5-cycle $C$ in $G$ is $S$-saturated if $|V(C) \cap S|=2$. For $x \in S$ we say that an $S$-saturated 5-cycle, $C$, is $x$-maximal if $x \in V(C)$ and $C$ is not contained in the interior of another $S$-saturated 5 -cycle whose vertices include $x$. (Note that the interior we use here is clearly not strict as $x$ does not lie in the strict interior of any $x$-maximal 5 -cycle.)

As we fix our attention on a particular vertex $x \in S$, we shall label the neighbours of $x$ by $x_{1}, x_{2}, \ldots, x_{k}$ as we scan anticlockwise about $x$. Since all vertices in $S$ have degree 5 or $6, k \in\{5,6\}$. 
(a)

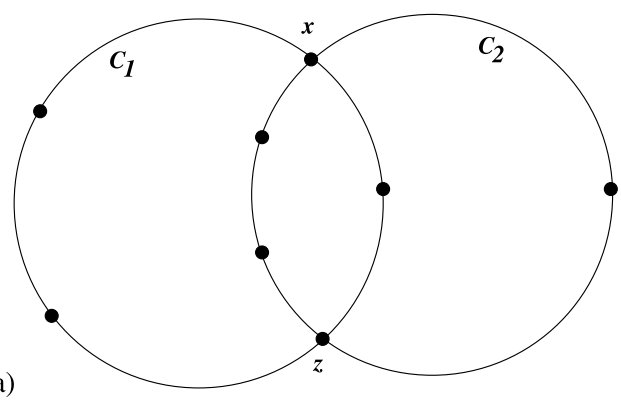

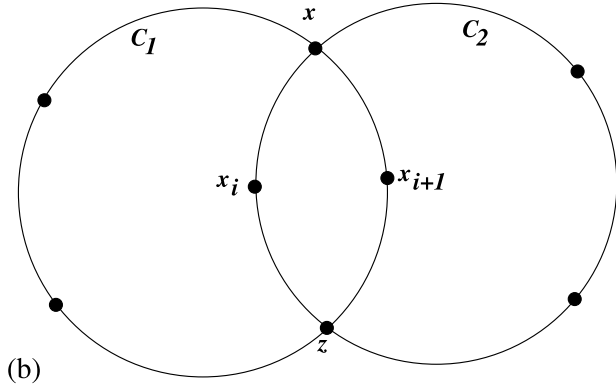

(b)

Fig. 1. Two $x$-maximal 5-cycles $C_{1}, C_{2}$ with overlapping strict interiors.

If, for each $x \in S, G$ has no $x$-maximal 5-cycle (i.e., there exists no $S$-saturated 5-cycle containing $x$ ), then $Q$ has no edge and hence is 0 -degenerate and has chromatic number 1. So we may assume that $G$ has at least one $x$-maximal 5-cycle for some $x \in S$.

It is possible that two $x$-maximal 5 -cycles have overlapping strict interiors. In Fig. 1 we have shown the two allowed ways in which two $S$-saturated 5 -cycles containing $x$ can have overlapping interiors. In Fig. 1 (a) we see that neither cycle $C_{1}$ nor $C_{2}$ can be $x$-maximal. Thus two $x$-maximal 5 -cycles with overlapping strict interiors must be as in Fig. 1 (b). Since there are no separating 4-cycles, the intersection of their strict interiors has no vertex of $G$. Moreover, $G$ cannot have the edge $x z$ and thus must have the edge $x_{i} x_{i+1}$. Consequently, the intersection of the strict interiors of two $x$-maximal 5 -cycles is either empty or the strict interior of a 4 -cycle of the form $x x_{i} z x_{i+1} x$ (traversed anticlockwise) where $x x_{i}, x x_{i+1}$ are consecutive edges incident with $x$ with respect to our fixed embedding of $G$ in the plane. Clearly, there is no third $x$-maximal 5 -cycle whose strict interior contains the edge $x_{i} x_{i+1}$ by the planarity. So, $x_{i} x_{i+1}$ is in the interior of at most two $x$-maximal 5 -cycles. (It is also clear that the edge $x_{i} x_{i+1}$ cannot be an edge in an $x$-maximal 5 -cycle.) Counting $x$-maximal 5 -cycles in this way, we count at most $6 \times 2=12 x$-maximal 5-cycles.

Now define $p(x)$ to be the number of those vertices $y$ of $S$ such that there exists an $x$-maximal 5-cycle having $y$ in its strict interior. Note that, if $x$ lies in no $x$-maximal 5 -cycle, then $p(x)=0$. Moreover, such a vertex $x$ has degree 0 in $Q$. Choose $x$ such that $p(x)$ is minimum.

If $p(x)=0$, then each neighbour of $x$ in $Q$ is on some $x$-maximal 5-cycle. Hence $x$ has at most 12 neighbours in $Q$ and we are done. So assume that $p(x)>0$.

Consider a vertex $y$ which contributes to $p(x)$. Then $y$ is strictly inside an $x$-maximal 5-cycle $C_{1}$. Let $C_{1}=x x_{1} v s x_{1+i} x$, where $i \geq 2, s \in S \backslash\{x\}$. Note, there is a path, $x_{1} x_{2} \ldots x_{1+i}$ joining $x_{1}$ to $x_{1+i}$ across the interior of $C_{1}$ such that the vertices in this path are all vertices in the neighbourhood of $x$. Thus $y$ is in the strict interior of $C_{1}^{\prime}=$ $x_{1} x_{2} \ldots x_{1+i} s v x_{1}$. The minimality of $p(x)$ implies that $p(y) \geq p(x)$ and thus there must be a $y$-maximal 5 -cycle $C_{2}$ extending beyond $C_{1}$. That is to say, $C_{2}$ must cross $C_{1}$. Moreover, $C_{2}$ must contain vertices in $S$ in its strict interior lying on or outside $C_{1}$. As such, $V\left(C_{2}\right)$ contains $y$, two vertices from $\left\{x_{1}, x_{1+i}, v, s\right\}$ and two further vertices $a, b$ 


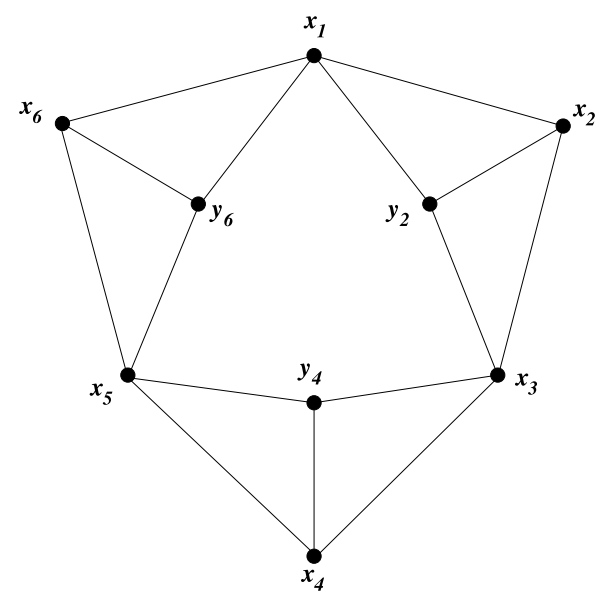

Fig. 2. A diamond-6-cycle with crucial and link vertices labelled according to the definition.

which lie outside $C_{1}$. (There cannot be only one vertex, say $a$, as $G$ has no separating 4-cycle. To see this, let us assume that $C_{2}$ consists of the path $\operatorname{vax}_{i+1}$, say, outside $C_{1}$ together with a path of length 3 inside $C_{1}$. Then either $v a x_{i+1} s v$ is a separating 4-cycle, which is a contradiction, or else there is an edge $v x_{i+1}$ which gives rise to another separating 4-cycle which is also a contradiction.) Since $y \in S$ and both vertices from $\left\{x_{1}, x_{1+i}, v, s\right\}$ must be neighbours of $y$ on $C_{2}, s \notin V\left(C_{2}\right)$. Similarly, since $i \geq 2, y$ cannot be adjacent to both $x_{1}$ and $x_{1+i}$ as then $y x_{1} x x_{1+i} y$ is a separating 4-cycle in $G$. Thus the neighbours of $y$ on $C_{2}$ are either $\left\{x_{1}, v\right\}$ or $\left\{v, x_{1+i}\right\}$. In the former case the strict interior of $C_{2}$ outside $C_{1}$ is bounded by the 4 -cycle $x_{1} v_{b a x_{1}}$ and thus cannot contain any vertex in $G$. In the latter case, the independence of $S$ together with the fact that $G$ is a triangulation requires that $v x_{1+i} \in E(G)$ but then $x x_{1} v x_{1+i} x$ is a separating 4-cycle in $G$; a contradiction. From this we conclude that no such $y$ exists and that we must have an $x \in S$ with $p(x)=0$. Consequently, $Q$ is 12-degenerate and hence of chromatic number at most 13.

We will also need to determine more about the distribution of vertices of degrees 5 and 6 with respect to certain separating 6-cycles. Again we introduce the following definitions and terminology to facilitate this investigation.

Definition. A diamond-6-cycle is a graph consisting of the cycles $x_{1} x_{2} x_{3} x_{4} x_{5} x_{6} x_{1}$ and $x_{1} y_{2} x_{3} y_{4} x_{5} y_{6} x_{1}$ and the edges $x_{2} y_{2}, x_{4} y_{4}, x_{6} y_{6}$, see Fig. 2. The vertices $x_{1}, x_{3}, x_{5}$ are called link vertices. The vertices $x_{2}, y_{2}, x_{4}, y_{4}, x_{6}, y_{6}$ are referred to as crucial vertices. We only consider a diamond-6-cycle drawn on a surface, and we assume that all its triangles are face boundaries. If a diamond-6-cycle is drawn as in Fig. 2, then $x_{2}, x_{4}, x_{6}$ are outer-crucial (because they are on the outer face boundary of the diamond-6-cycle) and $y_{2}, y_{4}, y_{6}$ are inner-crucial. 
With the labelling indicated in Fig. 2 we refer to the subgraph induced on $x_{1}, x_{2}, y_{2}, x_{3}$, as a diamond of the diamond-6-cycle. Clearly there are three diamonds in each diamond6 -cycle. The edge $x_{2} y_{2}$ is called the crucial axis of the diamond induced by $x_{1}, x_{2}, y_{2}, x_{3}$. Other crucial axes are defined similarly.

The subgraph induced on $x_{1}, x_{2}, y_{2}$ is referred to as a crucial triangle. Again it is clear that each diamond-6-cycle has six crucial triangles.

We make the following definition for general surfaces as we will use the generality later in the paper.

Definition. Let $G$ be a 5 -connected triangulation of surface $\Sigma$ and let $S \subseteq V(G)$ be an independent set of vertices each of which has degree 5 or 6 in $G$. The diamond auxiliary graph $\mathcal{D}_{S}(G)$ is the graph with vertices $V\left(\mathcal{D}_{S}(G)\right)=S$ with two vertices adjacent if they are crucial vertices in a common diamond-6-cycle in $G$ containing three vertices of $S$.

We now state a bound for the chromatic number of the diamond auxiliary graph for a 5-connected triangulation of the plane. This is a special case of Lemma 10 which is proved for general surfaces in Section 4.2.

Lemma 3. Let $G$ be a 5-connected plane triangulation, and let $S \subseteq V(G)$ be an independent set of vertices of degree 5 or 6 in $G$ such that no two vertices of $S$ are in a cycle of length at most 5. Then the diamond auxiliary graph $\mathcal{D}_{S}(G)$ is d-degenerate and hence has chromatic number at most $d+1$, where $d=300$.

One final configuration we would like to ensure does not occur is a 4-cycle containing two of our chosen vertices of degrees 5 and 6 . In the following lemma we are able to avoid such a situation while keeping at least one quarter of the chosen vertices. We also note that, having chosen a set of independent vertices so that no pair of them lies in a common 4-cycle, no triple can lie in the neighbourhood of a single vertex of degree 6 .

In Section 4.2 we will extend the following lemma to general surfaces in Lemma 8 but, because it is central to the development of our main result and the proof in the planar case is very short, we include a proof for this special case here.

Lemma 4. Let $G$ be a 5-connected plane triangulation. Let $S$ be an independent set of vertices of degree 5 or 6 . Then there is a subset $S^{\prime} \subseteq S$ with $\left|S^{\prime}\right| \geq|S| / 4$ such that no pair of vertices in $S^{\prime}$ lies in a common 4 -cycle in $G$.

Proof. Let $R$ be the graph defined on $S$ such that $s s^{\prime} \in E(R)$ (with $s, s^{\prime} \in S$ ) if and only if $s, s^{\prime}$ are on a common 4-cycle in $G$. Clearly, we can construct $R$ with an embedding inherited from $G$ such that $R$ is a plane graph. (For if $s s^{\prime} \in E(R)$, then there is a 4-cycle $s x s^{\prime} y s$. Since this is not a separating cycle, $G$ has the edge $x y$. We now replace $x y$ by $s s^{\prime}$.) As such, $R$ has chromatic number at most 4 , by the Four Color Theorem, and thus contains an independent set of size at least $|S| / 4$. 
As our final preliminary consideration, we wish to note that, in a 5-connected planar triangulation, we can delete a collection of edges incident with vertices in a specified set and leave the resulting graph 4-connected. Lemma 5 is a special case of Lemma 11 proved later in Section 4.2.

Lemma 5. Let $G$ be a 5-connected plane triangulation, and let $S \subseteq V(G)$ be an independent set of vertices of degrees 5 or 6 such that no two vertices in $S$ lie in a common 4-cycle or 5-cycle, and no triple of vertices of $S$ are all crucial vertices in a common diamond-6-cycle. Let $F \subseteq E(G)$ be chosen so that $|F|=|S|$ and for each vertex $v \in S$ there is precisely one edge in $F$ incident with $v$. Then the graph $G^{\prime}=G-F$ is 4-connected.

\section{Hamiltonian cycles in planar triangulations}

Theorem 2. Every 5-connected planar triangulation with $n$ vertices contains at least $1.000003^{n}$ Hamiltonian cycles.

Proof. Let $G$ be a 5 -connected planar triangulation on $n$ vertices. By Theorem $1, G$ has more than one Hamiltonian cycle. So Theorem 2 holds for small values of $n$. Let $T \subseteq V(G)$ be the set of vertices of degree 5 or 6 . Since $G$ is 5 -connected and planar, $\delta(G)=5$ and, since $G$ is also a triangulation, by Euler's formula, $|E(G)|=3 n-6$, so the average degree is less than 6 . Consequently $|T|>n / 2$.

By the Four Color Theorem, $G$ is 4-colorable and hence $T$ contains an independent set of size at least $n / 8$. Let $T^{\prime}$ be such a set.

Now, by Lemma 2, we can choose a subset $T^{\prime \prime} \subseteq T^{\prime}$ such that no two vertices in $T^{\prime \prime}$ lie in a common 5-cycle and such that $\left|T^{\prime \prime}\right| \geq\left|T^{\prime}\right| / 13 \geq n / 104$.

We consider now the diamond auxiliary graph $D_{T^{\prime \prime}}(G)$. By Lemma $3, D_{T^{\prime \prime}}(G)$ has chromatic number at most 301 and thus we may choose a colour class, $T^{\prime \prime \prime}$, with at least $\left|V\left(D_{T^{\prime \prime}}(G)\right)\right| / 301$ vertices.

The set $T^{\prime \prime \prime}$ may still contain pairs of vertices both of which lie in a common 4-cycle in $G$. By Lemma 4 , there is a subset $S \subseteq T^{\prime \prime \prime}$, with $|S| \geq\left|T^{\prime \prime \prime}\right| / 4 \geq n /(104 \times 301 \times 4)=$ $n / 125216$ in which no such pair exists.

Now we construct a graph $G^{\prime}$ from $G$ by randomly deleting one edge incident with each vertex in $S$ (determined above).

The graph $G^{\prime}$ so formed remains planar (since $G$ is), is 4-connected by Lemma 5 and each vertex in $S$ has degree either 4 or 5 . Thus, by Theorem $1, G^{\prime}$ contains a Hamiltonian cycle.

Let us suppose that there are $n_{1}$ vertices in $S$ with degree 5 in $G$ and $n_{2}$ with degree 6 (so $n_{1}+n_{2}=|S|$ ). Then there are $5^{n_{1}} \times 6^{n_{2}}$ ways to form $G^{\prime}$ from $G$ and the same cycle through $S$ may be contained in $3^{n_{1}} \times 4^{n_{2}}$ of these. Thus there are at least $\left(5^{n_{1}} \times\right.$ $\left.6^{n_{2}}\right) /\left(3^{n_{1}} \times 4^{n_{2}}\right) \geq(5 / 3)^{n_{1}} \times(6 / 4)^{n_{2}} \geq(3 / 2)^{|S|} \geq(3 / 2)^{n / 125216} \geq 1.000003^{n}$ Hamiltonian cycles in $G$. 
This completes the proof.

\section{Other surfaces}

\subsection{Large face-width}

The techniques applied in the previous section can be applied to surfaces of higher genus with only minor modifications. In particular, Lemmas 1-5 hold unchanged for 5 -connected triangulations of surfaces of higher genus if we impose a requirement that the face-width of our graph is sufficiently large. For example, if we require that face-width is at least 7 , then all separating 5-cycles and 6-cycles separate the surface into a disc and a space which is not a disc. By focusing on the disc, we obtain the variations of Lemmas 1-5 required. Of course, some of the constants determined to bound the size of our special set of vertices will change (for example, projective planar triangulations may have chromatic number 6 , toroidal triangulations 7 and so on), but will still be a constant proportion of the number of vertices in our 5-connected triangulation.

On the projective plane we have the following theorem due to Thomas and $\mathrm{Yu}$ [11].

Theorem 3 ([11]). Let $G$ be a 4-connected projective planar graph. Then $G$ is Hamiltonian.

Consequently, we can establish an analogous result to Theorem 2 for the projective plane.

Theorem 4. Every 5-connected projective planar triangulation with $n$ vertices and facewidth at least 7 has exponentially many Hamiltonian cycles.

As we later prove a stronger result, without the face-width condition, we omit the details of the proof of Theorem 4.

For the torus there is a long standing conjecture due to Nash-Williams [10] that every 4-connected toroidal graph is Hamiltonian. The analogous problem for the Klein bottle is also open. If this is true, then again, with slight modification to the counting (e.g. a triangulation of the torus (or Klein bottle) may have chromatic number 7 (or 6) rather than 4 etc.) and with the addition of the requirement of face-width at least 7 , we could conclude that every 5-connected triangulation of the torus or Klein bottle has exponentially many Hamiltonian cycles. Again, a stronger result follows from the general treatment in Section 4.2 .

We can make use of Theorem 5 below by Böhme, Mohar and Thomassen [7] to show that for any surface, a 5-connected triangulation of suitably large face-width on that surface contains exponentially many cycles. Since the Euler genus appears in this and several subsequent results, we include a definition here. For more details the interested reader should see [9, page 101$]$. 
Definition. Let $\Sigma$ be a surface with Euler characteristic $\chi(\Sigma)$. Then the Euler genus, $e g(\Sigma)=2-\chi(\Sigma)$

Theorem 5 ([7]). There is a function $a: \mathbb{N}_{0} \times \mathbb{R}_{+} \longrightarrow \mathbb{N}$ such that for every $\epsilon>0$ and every 4-connected graph that has an embedding of Euler genus eg and face-width at least $a(e g, \epsilon)$, there are two cycles $C_{1}, C_{2}$ in $G$ such that

(1) $V\left(C_{1}\right) \cup V\left(C_{2}\right)=V(G)$, and

(2) $\left|V\left(C_{i}\right)\right| \geq(1-\epsilon)|V(G)|$, for $i=1,2$.

Theorem 6. For each surface of Euler genus eg, there is a constant $\alpha(e g)>1$, such that every 5 -connected triangulation $G$ with $n$ vertices of face-width $2 a(e g, 1 / 2)$ has at least $(\alpha(e g))^{n}$ cycles.

Proof. Clearly, $G$ has more than one cycle. We choose $\alpha(e g)$ so close to 1 that the inequality $(\alpha(e g))^{n}>2$ implies that $n$ is large compared to eg. By Euler's formula the number of edges in $G$ is at most $3 n-6+3 \mathrm{eg}$. Hence the average degree is at most $6+(6 e g-12) / n$ which is close to 6 . Since the minimum degree is at least 5 , it follows that almost half the vertices have degree 5 or 6 . We repeat the proof of Theorem 2 but here, after deleting precisely one edge from each of our selected vertices, we have a 4 -connected graph of face-width at least $a(e g, 1 / 2)$ on our surface (because the deleted edges form a star forest). Thus we can apply Theorem 5 to find that there are two long cycles $\left(C_{1}, C_{2}\right)$ covering the entire vertex set of $G$. Following the analysis in Theorem 2, we get a constant $\beta>1$ such that there are at least $\beta^{n}$ pairs of such cycles in $G$. Now, with $\alpha(e g)=\sqrt{\beta}$, the result follows.

\subsection{The projective plane, the torus and the Klein bottle}

We shall now extend Theorem 2 to the projective plane and, subject to Nash-Williams' conjecture for the torus and its analogue for the Klein bottle, to the torus and Klein bottle. For this we need generalisations of Lemmas 1-5 for surfaces other than the plane without the addition of a minimal face-width condition. Some of these may be of independent interest. For example, if a graph $G$ is embedded on a surface, we may define a new graph $H$ such that two vertices in $H$ are neighbours if they lie on a common 5-cycle in $G$. If $G$ is 5 -connected, then the chromatic number is bounded above by a function of the Euler genus of $G$. This is not true if $G$ is 4 -connected. Since face-width restrictions are still required to apply Theorem 5 , these generalisations will be of most immediate interest in the case of the projective plane, torus and Klein bottle.

Definition. Let $\Sigma$ be a surface and let $K$ be a set of points on $\Sigma$. A simple closed curve $C$ is said to be $K$-contractible if $C$ is contractible in the usual topological sense and, in addition, the interior of $C$ contains no point in $K$. 
Note: $\emptyset$-contractibility is just the usual contractibility.

Definition. Let $\Sigma$ be a surface and let $\mathcal{C}$ be a set of simple closed curves on $\Sigma$. A contractible curve $C \in \mathcal{C}$ is called maximal if there is no other curve $C^{\prime} \in \mathcal{C}$ such that $C$ is contained in the interior of $C^{\prime}$.

Definition. A cylinder between simple closed curves $C_{1}$ and $C_{2}$ is an annulus bounded by $C_{1}$ and $C_{2}$.

Definition. A contracted cylinder between simple closed curves $C_{1}$ and $C_{2}$ is obtained from the cylinder between simple closed curves $C_{1}$ and $C_{2}$ by taking a simple curve through the interior of the annulus joining a point on $C_{1}$ and a point on $C_{2}$ and contracting it to a single point now common to both curves.

A cylinder or contracted cylinder may contain its boundary in which case it is closed. It may also be disjoint from its boundary in which case it is open.

Lemma 6. Let $K$ be a non-empty set of points in the plane with $|K|=k$. Suppose $\mathcal{C}$ is a set of simple closed curves in the plane, none of which intersects $K$, and with the property that for each curve $C \in \mathcal{C}$, at most one point in $C$ can be contained in other curves in $\mathcal{C}$. Suppose further that each curve in $\mathcal{C}$ is not $K$-contractible (that is, each curve has a point from $K$ in its interior). Then, whenever $c=|\mathcal{C}| \geq 2 k$, we have an open cylinder or contracted cylinder bounded by two curves in $\mathcal{C}$ and containing no point in a curve of $\mathcal{C}$ and no point in $K$.

Proof. Let $K, k, \mathcal{C}$ and $c$ be as in the statement of the lemma. We assume that $c \geq 2 k$ and proceed by induction on $k$.

Clearly, when $k=1$, if we have two or more curves in $\mathcal{C}$, then the single point in $K$ must lie in the interior of all curves in $\mathcal{C}$ which are "stacked" concentrically around it. The desired cylinder or contracted cylinder is then immediately apparent.

We say that a simple closed curve $C \in \mathcal{C}$ is maximal in $\mathcal{C}$ if it does not lie entirely inside the closed disc bounded by any other simple closed curve in $\mathcal{C}$. Let the simple closed curves $C_{1}, C_{2}, \ldots, C_{m}$ be maximal in $\mathcal{C}$, let $k_{i}$ be the number of points of $K$ in the interior of $C_{i}$ and let $c_{i}$ be the number of simple closed curves from $\mathcal{C}$ inside $C_{i}$, including $C_{i}$ itself, $1 \leq i \leq m$.

Assume first that $m \geq 2$. If for some $i, 1 \leq i \leq m$, we have $c_{i} \geq 2 k_{i}$, we have the desired cylinder or contracted cylinder by induction. So suppose this does not happen. That is $c_{i} \leq 2 k_{i}-1$ for each $1 \leq i \leq m$. Hence,

$$
c=\sum_{i=1}^{m} c_{i} \leq \sum_{i=1}^{m}\left(2 k_{i}-1\right) \leq 2 k-m \leq 2 k-2 .
$$

This contradicts our assumption that $c \geq 2 k$ and thus establishes the result. 
Assume next that $m=1$. Let $C_{1}^{\prime}, C_{2}^{\prime}, \ldots, C_{q}^{\prime}$ be the maximal curves in $\mathcal{C} \backslash C_{1}$. If $K$ has a point $p$ outside each of $C_{1}^{\prime}, C_{2}^{\prime}, \ldots, C_{q}^{\prime}$, then we apply induction to $\mathcal{C} \backslash\left\{C_{1}\right\}$ and $K \backslash\{p\}$ to obtain the result.

If $K$ has no point outside each of $C_{1}^{\prime}, C_{2}^{\prime}, \ldots, C_{q}^{\prime}$, and $q \geq 2$, then we repeat the argument in the case $m \geq 2$ to $\mathcal{C} \backslash\left\{C_{1}\right\}$ and obtain a contradiction.

If $q=1$ and $K$ has no point outside $C_{1}^{\prime}$, then the annulus between $C_{1}$ and $C_{1}^{\prime}$ is the desired cylinder or contracted cylinder.

Lemma 7. Let $\Sigma$ be a surface of Euler genus eg $(\Sigma)$. Suppose that, on $\Sigma$, we have a set $K$ of $|K|=k \geq 0$ points, and suppose $\mathcal{C}$ is a set of simple closed non-K-contractible curves on $\Sigma$, none of which intersects $K$, and with the property that for each curve $C \in \mathcal{C}$, at most one point in $C$ can be contained in other curves in $\mathcal{C}$. If $c=|\mathcal{C}| \geq 2 k+5 \operatorname{eg}(\Sigma)$, then there is either a cylinder or a contracted cylinder bounded by two of the simple closed curves in $\mathcal{C}$ such that the (contracted) cylinder contains none of the points in $K$.

Proof. We proceed by induction on the Euler genus, $e g(\Sigma)$ of $\Sigma$.

When $e g(\Sigma)=0$, Lemma 6 applies and the result follows.

Assume the result is true for $e g(\Sigma) \leq n$ and consider $e g(\Sigma)=n+1$. We shall always assume that $c \geq 2 k+5 \operatorname{eg}(\Sigma)$.

Case 1: Suppose all curves in $\mathcal{C}$ are $\emptyset$-contractible. Then we apply the proof of Lemma 6 to obtain the desired result.

Case 2: Suppose there is a one-sided non-contractible curve $C \in \mathcal{C}$ and suppose $p \in C$ also belongs to curves $C_{1}, \ldots, C_{t} \in \mathcal{C}$. If $t=0$, then $p$ is any point on $C$. We cut along $C$, cap with a disc and add a line across the cap joining both copies of $p$ resulting from the cut along $C$. Now contract this line to form two contractible copies of $C$ having $p$ in common. Form $K^{\prime}$ by adding a new point to $K$ in the interiors of each of the new contractible copies of $C$ and then discard both copies of $C$ to form $\mathcal{C}^{\prime}$. The new surface, $\Sigma^{\prime}$, has $e g\left(\Sigma^{\prime}\right)=e g(\Sigma)-1$, set of $k+2$ points $K^{\prime}$ and curve set $\mathcal{C}^{\prime}$ complying with the statement of the lemma. Moreover,

$\left|\mathcal{C}^{\prime}\right|=c-1 \geq(2 k+5 \operatorname{eg}(\Sigma))-1=(2 k+5(n+1))-1=2(k+2)+5 n=2\left|K^{\prime}\right|+5 e g\left(\Sigma^{\prime}\right)$.

By our inductive hypothesis, we have the desired cylinder or contracted cylinder on $\Sigma^{\prime}$ and this lifts to $\Sigma$, as required.

Case 3: $\mathcal{C}$ contains a two-sided non-contractible non-separating curve $C$. Again, we cut along $C$, this time producing two copies of $C$, call them $C$ and $C^{\prime}$, each bounding a "hole". Cap each resulting "hole" with a disc, adding new special points $p, p^{\prime}$, one in the interior of each cap. It is possible that there is a curve $C^{\prime \prime} \in \mathcal{C}$ sharing a point $q$ with $C$ and that $C^{\prime \prime}$ becomes an open curve joining $q$, in $C$, to its copy, $q^{\prime}$, in $C^{\prime}$. Of course, there may be more than one such curve but we focus on $C^{\prime \prime}$. Now contract $C^{\prime \prime}$ to identify $q$ and $q^{\prime}$. We shall keep the label $q$ for this point. All other curves in $\mathcal{C}$ sharing $q$ then form curves 
sharing the new point labelled $q$. The new surface, $\Sigma^{\prime}$, has $e g\left(\Sigma^{\prime}\right)=e g(\Sigma)-2$. On this new surface, we have a set $K^{\prime}=K \cup\left\{p, p^{\prime}\right\}$ of $k^{\prime}=k+2$ points and a set $\mathcal{C}^{\prime}$ of $c^{\prime}=c$ or $c^{\prime}=c+1$ curves which pairwise share at most one point and are all non- $K^{\prime}$-contractible unless one of them, say $C^{\prime \prime \prime}$, together with $C^{\prime \prime}$ form a desired contracted cylinder.

Suppose therefore that all curves in $\mathcal{C}^{\prime}$ are non- $K^{\prime}$-contractible and thus

$$
c^{\prime} \geq c \geq 2 k+5 \operatorname{eg}(\Sigma)=2 k^{\prime}-4+5 \operatorname{eg}\left(\Sigma^{\prime}\right)+10=2 k^{\prime}+5 \operatorname{eg}\left(\Sigma^{\prime}\right)+6 .
$$

By our inductive hypothesis, we have the desired cylinder or contracted cylinder on $\Sigma^{\prime}$ and this lifts to $\Sigma$, as required.

Case 4: Finally, we may assume that $\mathcal{C}$ contains a (two-sided) non-contractible separating curve $C$. Once more, we cut along $C$ leaving one copy of $C$ on each side of the cut. Again, we cap each "hole" with a disc. This results in two surfaces, neither of which is the sphere. Call these new surfaces $\Sigma_{1}, \Sigma_{2}$. Note that, for each $i=1,2$, in $\Sigma_{i}$, the copy of $C$ bounding the capping disc is contractible and $K$-contractible. If any other curve $C^{\prime}$ in $\mathcal{C} \cap \Sigma_{i}$ is $K$-contractible in $\Sigma_{i}$, then $C$ and $C^{\prime}$ bound a desired cylinder in $\Sigma$. So we assume that no such $C^{\prime}$ exists in either $\Sigma_{i}$, and we discard the two copies of $C$. Now we have surfaces $\Sigma_{1}, \Sigma_{2}$ with special point sets, $K_{1}, K_{2}$ respectively such that $e g\left(\Sigma_{1}\right)+e g\left(\Sigma_{2}\right)=e g(\Sigma)$ and $\left|K_{1}\right|+\left|K_{2}\right|=k_{1}+k_{2}=k$. Also, with the obvious modifications we form appropriate sets of curves $\mathcal{C}_{1}$ and $\mathcal{C}_{2}$, so that setting $c_{i}=\left|\mathcal{C}_{i}\right|$ we have $c_{1}+c_{2}=c-1$ since no two curves in $\mathcal{C}$ intersect twice. By induction, if, for either value of $i, c_{i} \geq 2 k_{i}+5 e g\left(\Sigma_{i}\right)$, we have a cylinder or contracted cylinder on $\Sigma_{i}$ that lifts to the desired cylinder or contracted cylinder on $\Sigma$. So assume this is not the case. That is

$$
\begin{aligned}
c=c_{1}+c_{2}+1 & \leq\left(2 k_{1}+5 \operatorname{eg}\left(\Sigma_{1}\right)-1\right)+\left(2 k_{2}+5 \operatorname{eg}\left(\Sigma_{2}\right)-1\right)+1 \\
& =2 k+5 \operatorname{eg}(\Sigma)-2+1 \\
& =2 k+5 \operatorname{eg}(\Sigma)-1 .
\end{aligned}
$$

This contradicts our assumption that $c \geq 2 k+5 \operatorname{eg}(\Sigma)$ and the result follows.

Corollary 1. Let $\Sigma$ be a surface of Euler genus eg $(\Sigma)$ and let $\mathcal{C}$ be a set of simple closed curves on $\Sigma$ with the property that for each curve $C \in \mathcal{C}$, at most one point in $C$ can be contained in other curves in $\mathcal{C}$. Then,$|\mathcal{C}| \geq 5$ eg $(\Sigma)$ implies there are two homotopic curves in $\mathcal{C}$.

Corollary 2. Let $r \geq 1$ be an integer, let $\Sigma$ be a surface of Euler genus eg $(\Sigma)$ and let $\mathcal{C}$ be a set of simple closed curves on $\Sigma$ with the property that for each curve $C \in \mathcal{C}$, at most one point in $C$ can be contained in other curves in $\mathcal{C}$. Then, $|\mathcal{C}| \geq(r-1) 5 \operatorname{eg}(\Sigma)+1$ implies there are $r$ homotopic curves in $\mathcal{C}$. 
Returning our focus to graphs on surfaces and, in particular, our consideration of 5 -connected triangulations of surfaces we present a lemma with which we may restrict our set of special vertices so that no two lie in a common 4-cycle.

Lemma 8. Let $G$ be a 5-connected triangulation of surface $\Sigma$ of Euler genus eg $(\Sigma)=\sigma$ and let $S \subseteq V(G)$ be an independent set of vertices of degree 5 or 6 . Let $Q$ be the graph with $V(Q)=S$ such that two vertices are neighbours in $Q$ iff they lie on a common 4-cycle in $G$. Then $Q$ is q-degenerate and therefore has chromatic number at most $q+1$, where $q=\left(\begin{array}{l}6 \\ 2\end{array}\right)(10 \sigma+1)$.

Proof. We wish to show that $Q$ is $q$-degenerate. Clearly, if $\Delta(Q) \leq q$, we are done, and so we consider a vertex $v \in S=V(Q)$ with degree greater than $q$ in $Q$. Thus there are vertices $v_{1}, v_{2}, \ldots, v_{q+1} \in S=V(Q)$ such that $v, v_{i}$ lie on a common 4-cycle in $G$, $1 \leq i \leq q+1$. Of these 4 -cycles, at least $(q+1) /\left(\begin{array}{l}6 \\ 2\end{array}\right)$ include a common path uvw through $v$, since $v$ has degree 5 or 6 in $G$. Relabelling if necessary, we have $u v w v_{i} u$ is a 4 -cycle containing $v$ and $v_{i}$ for $1 \leq i \leq q+1$. But by Corollary 2, there must be at least three homotopic 4-cycles through uvw and hence a 4-cut in $G$. (To see this, we contract the path uvw into a single vertex and apply Corollary 2 to the resulting double edges.) This contradiction establishes the result.

We can now determine the following generalisation of Lemma 2 for other surfaces.

Lemma 9. Let $G$ be a 5-connected triangulation of surface $\Sigma$ of Euler genus eg $(\Sigma)=\sigma$ and let $S$ be an independent set of vertices of degree 5 or 6 . Assume that no two vertices of $S$ lie on a common 4-cycle. Let $P$ be the graph defined on $S$ such that two vertices are neighbours in $P$ iff they lie on a common 5-cycle in $G$. Then $P$ is p-degenerate and therefore has chromatic number at most $p+1$, where $p=\left(\begin{array}{l}6 \\ 2\end{array}\right)(40 \sigma+1)$.

Proof. We wish to show that $P$ is $p$-degenerate. Clearly, if $\Delta(P) \leq p$, we are done so consider a vertex $v \in S=V(P)$ with degree greater than $p$ in $P$. Then $v$ lies on at least $(p+1) 5$-cycles in $G$, each of which contains precisely one other vertex in $S$. Of these, at least $(p+1) /\left(\begin{array}{l}6 \\ 2\end{array}\right)$ include a common path uvw through $v$. Thus, by Corollary 2 there are at least nine homotopic 5-cycles that share the path uvw. (Again, we contract uvw. Two of the resulting 3-cycles have only the contracted vertex in common because no two vertices in $S$ lie on a common 4-cycle.) Consequently we have nine 5-cycles $C_{i}=u v w x_{i} y_{i} u$, $1 \leq i \leq 9$, and a disc bounded by the cycle $C_{v}=w x_{1} y_{1} u y_{9} x_{9} w$ (assuming our labelling is such that all $x_{i}, y_{i}$ are on or inside $C_{v}$ ). We say that $v$ captures all those vertices in $S$ that lie in the closed disc bounded by $C_{v}$. Let us suppose that $v$ and the associated nine 5 -cycles have been chosen to minimise the number of vertices in $S$ captured by $v$. Now consider the 5 -connected plane triangulation $G^{\prime}$ obtained from $G$ by taking the plane subgraph of $G$ bounded by the 6-cycle $C_{v^{\prime}}=w x_{4} y_{4} u y_{6} x_{6} w$ and adding a new vertex $v^{\prime}$ adjacent to each of the six vertices in $V\left(C_{v^{\prime}}\right)$. The associated graph $P^{\prime}$ defined as 
$V\left(P^{\prime}\right)=S \cap V\left(G^{\prime}\right)$ and two vertices are adjacent iff they lie in a common 5 -cycle in $G^{\prime}$ contains a vertex $v^{\prime \prime}$ with $\operatorname{deg}_{P^{\prime}}\left(v^{\prime \prime}\right) \leq 12$ by Lemma 2 .

Claim: The vertex $v^{\prime \prime}$ has $\operatorname{deg}_{P}\left(v^{\prime \prime}\right) \leq p$.

To see this we note that all 5-cycles in $G$ containing $v^{\prime \prime}$ must lie within the disc bounded by $C_{v}$ unless such a cycle passes through $u, w$ and one further vertex in $S$ outside the disc bounded by $C_{v}$. By Corollary 2 here can be at most $10 \sigma$ such 5 -cycles without giving rise to a separating 4 -cycle in $G$. So we may assume that $v^{\prime \prime}$ lies on at least $((p+1)-10 \sigma-12) 5$-cycles which extend beyond $C_{v^{\prime}}$ but are wholly contained within the disc bounded by $C_{v}$, and each contains another vertex in $S$. In particular, there are neighbours $u^{\prime \prime}, w^{\prime \prime}$ of $v^{\prime \prime}$ such that there are at least $((p+1)-10 \sigma-12) /\left(\begin{array}{l}6 \\ 2\end{array}\right) \geq 9$ 5 -cycles through $u^{\prime \prime} v^{\prime \prime} w^{\prime \prime}$, each containing a vertex in $S$ other than $v^{\prime \prime}$. Since all of these 5 -cycles are homotopic, and all of the vertices in $S$ captured by $v^{\prime \prime}$ form a proper subset of those captured by $v$, we have a contradiction to the minimality of $v$. The claim and result now follow.

We now bound the chromatic number of the diamond auxiliary graph for a 5-connected triangulation of any surface. Note that Lemma 3 stated earlier is just the planar case of the following lemma.

Lemma 10. Let $G$ be a 5-connected triangulation of surface $\Sigma$ of Euler genus eg $(\Sigma)=\sigma$ and let $S \subseteq V(G)$ be an independent set of vertices of degree 5 or 6 in $G$ such that no two are in a cycle of length at most 5. Then the diamond auxiliary graph $\mathcal{D}_{S}(G)$ is $d$-degenerate and hence has chromatic number at most $d+1$, where $d=300(\sigma+1)$.

Proof. The proof follows a now familiar pattern. First we note that if every vertex in $\mathcal{D}_{S}(G)$ has degree at most $d$, then we are done. So we may suppose that some vertex $v \in S=V\left(\mathcal{D}_{S}(G)\right)$ has degree at least $d+1$ in $\mathcal{D}_{S}(G)$. Since $v$ is a crucial vertex in at most six diamonds, at least $(d+1) / 6$ of the vertices adjacent to $v$ lie in diamond-6-cycles that include one of these diamonds in particular. We shall call this diamond $D_{0}$.

Each diamond-6-cycle containing $D_{0}$ contains three link vertices; two of those, $u_{0}, w_{0}$ are associated with $D_{0}$ and the third is not in $D_{0}$. Now there are at least $50(\sigma+1)$ distinct vertices $x_{i}, 1 \leq i \leq 50(\sigma+1)$, which are link vertices of diamond-6-cycles containing $D_{0}$, different from $u_{0}, w_{0}$. We consider a path of length 4 in each of these diamond-6-cycles joining $u_{0}, w_{0}$ and containing two vertices of $S$. Each of these paths can be extended to a cycle by adding a path in $D_{0}$ from $u_{0}$ to $w_{0}$. We may contract this path in $D_{0}$ to a vertex (and also contract any other edge which is incident with $u_{0}$ or $w_{0}$ and belongs to two of the above paths of length 4) and apply Corollary 2 to conclude that among these cycles there are at least eleven which are homotopic. As such, we have a disc, $L$, bounded by two of these paths between the link vertices associated with diamond $D_{0}$, containing nine further paths between the same two points. We say that the vertices in $S$ inside $L$ are captured by $D_{0}$ and these eleven diamond-6-cycles. 
We choose $D_{0}$ and the eleven homotopic diamond-6-cycles containing it to minimise the number of vertices in $S$ captured by $D_{0}$. Now we concentrate on the diamond-6-cycle corresponding to the middle one of these eleven passing through $L$. In particular, we focus on a crucial vertex $z \neq v$ in this diamond-6-cycle. If $\operatorname{deg}_{\mathcal{D}_{S}(G)}(z) \leq d$, we are done so suppose $\operatorname{deg}_{\mathcal{D}_{S}(G)}(z) \geq d+1$. First note that $z$ cannot be the crucial vertex in a diamond with link vertices $u_{0}, w_{0}$ as that would imply a 4-cycle containing $z$ and the vertex $v$ of $S$ in $D_{0}$. Now $\operatorname{deg}_{\mathcal{D}_{S}(G)}(z) \geq d+1$ and $\operatorname{deg}_{G}(z) \leq 6$, so $z$ lies in at most 6 diamonds and one of these, $D_{z}$, say, lies in at least $(d+1) / 12$ diamond-6-cycles. All of these are contained in $L$, since otherwise there would be a 4-cycle containing two vertices of $S$, a contradiction. Thus, there are eleven homotopic diamond-6-cycles through $D_{z}$ inside $L$, and there is a disc, $L_{z}$ formed in the same way as we formed $L$ from the eleven homotopic diamond-6-cycles through $D_{0}$. But this implies that there are strictly fewer vertices in $S$ captured by $D_{z}$ and its eleven diamond-6-cycles than are captured by $D_{0}$ and its eleven diamond-6-cycles; a contradiction.

Lemma 5 can now be generalised as follows.

Lemma 11. Let $G$ be a 5-connected triangulation of surface $\Sigma$ of Euler genus eg $(\Sigma)=\sigma$ and let $S \subseteq V(G)$ be an independent set of vertices of degree 5 or 6 in $G$ such that no two vertices in $S$ lie in a common 4-cycle or 5-cycle and no triple of vertices of $S$ are all crucial vertices in a common diamond-6-cycle. Let $F \subseteq E(G)$ be chosen so that $|F|=|S|$ and for each vertex $v \in S$, there is precisely one edge in $F$ incident with $v$. Then the graph $G^{\prime}=G-F$ is 4-connected.

Proof. Suppose to the contrary that $G^{\prime}$ contains a minimal separating vertex set, $K \subset$ $V\left(G^{\prime}\right)=V(G)$ such that $|K| \leq 3$.

Each component $A$ of $G^{\prime}-K$ has at least $5-|K|$ edges from $F$ joining it to other components of $G^{\prime}-K$. Each such edge, $f$, lies in the boundaries of two triangular faces in $G$, so each end vertex of $f$ is adjacent to two vertices in $K$. Thus $|K| \geq 2$. Moreover, each edge in $F$ has one end vertex in $S$, so if $f, f^{\prime}$ are edges in $F$ joining $A$ to other components in $G^{\prime}-K$, then there are end vertices $s, s^{\prime}$ of $f, f^{\prime}$ respectively in $S$. Thus, if $|K|=2$, then both $s$ and $s^{\prime}$ are adjacent to both vertices in $K$ which would require that two vertices in $S$ belong to a common 4-cycle. So we may assume $|K|=3$.

Again, let $A$ be a component of $G^{\prime}-K$ and let $F^{\prime} \subseteq F$ be the set of those edges in $F$ with precisely one end vertex in $A$. Each edge $f \in F^{\prime}$ lies in the boundaries of two triangular faces in $G$. The remaining vertex in each such triangular face lies in $K$. Since $|K|=3$, if there are four or more edges in $F^{\prime}$, then there must be two, $f, f^{\prime}$, say, such that the two neighbours of the ends of $f$ in $K$ are the same as for $f^{\prime}$. Again, this requires that two of the vertices in $S$ belong to a common 4-cycle. Hence either $\left|F^{\prime}\right|=2$ or $\left|F^{\prime}\right|=3$.

For each edge $e \in F^{\prime}$, we delete $e$ and replace it by an edge $e^{\prime}$ joining two vertices in $K$ such that we get a new triangulation. In this new triangulation $K$ is a separating set 
and hence $K$ induces a triangle by the observation following Lemma 1 . Thus in the case $\left|F^{\prime}\right|=2$ we have a separating 5 -cycle in $G$ that contains two vertices in $S$, contradicting the choice of $S$.

The case $\left|F^{\prime}\right|=3$ yields either a diamond-6-cycle with three vertices in $S$ as crucial vertices or $A$ is a single vertex of degree 6 with three neighbours in $S$ (and hence two of these vertices in $S$ lie on a common 4-cycle). Again, this is a contradiction and the result follows.

It is now an easy matter to apply the same analysis as in Section 3 to determine the following generalisation of Theorem 4.

Theorem 7. There exists a real number $\alpha>1$ such that every 5-connected projective planar triangulation with $n$ vertices has at least $\alpha^{n}$ Hamiltonian cycles.

Extending to the torus and Klein bottle we have the following.

Theorem 8. Suppose every 4-connected toroidal graph is Hamiltonian. Then, there exists a real number $\alpha>1$ such that every 5-connected toroidal triangulation with $n$ vertices has at least $\alpha^{n}$ Hamiltonian cycles.

Theorem 9. Suppose every 4-connected Klein bottle graph is Hamiltonian. Then, there exists a real number $\alpha>1$ such that every 5-connected triangulation of the Klein bottle with $n$ vertices has at least $\alpha^{n}$ Hamiltonian cycles.

\section{Acknowledgment}

This paper was initiated during Thomassen's visit to King Abdulaziz University in February 2014 under the Highly Cited Program. The paper was completed during Aldred's visit to the Technical University of Denmark August - October 2015 supported in part by the ERC Advanced Grant GRACOL.

\section{References}

[1] A. Alahmadi, R.E.L. Aldred, R. dela Cruz, P. Solé, C. Thomassen, The maximum number of minimal codewords in long codes, Discrete Appl. Math. 161 (2013) 424-429.

[2] A. Alahmadi, R.E.L. Aldred, R. dela Cruz, P. Solé, C. Thomassen, The maximum number of minimal codewords in an [n,k]-code, Discrete Math. 313 (2013) 1569-1574.

[3] A. Alahmadi, R.E.L. Aldred, R. dela Cruz, S. Ok, P. Solé, C. Thomassen, The minimum number of minimal codewords in an [n,k]-code and in graphic codes, Discrete Appl. Math. 184 (2015) 32-39.

[4] R.E.L. Aldred, C. Thomassen, On the number of cycles in 3-connected cubic graphs, J. Combin. Theory Ser. B 71 (1997) 79-84.

[5] C.A. Barefoot, L. Clark, R.C. Entringer, Cubic graphs with the minimum number of cycles, Congr. Numer. 53 (1986) 49-62.

[6] T. Böhme, J. Harant, M. Tkáč, On certain Hamiltonian cycles in planar graphs, J. Graph Theory 32 (1999) 81-96.

[7] T. Böhme, B. Mohar, C. Thomassen, Long cycles in graphs on a fixed surface, J. Combin. Theory Ser. B 85 (2002) 338-347. 
[8] S.L. Hakimi, E.F. Schmeichel, C. Thomassen, On the number of Hamiltonian cycles in a maximal planar graph, J. Graph Theory 3 (1979) 365-370.

[9] B. Mohar, C. Thomassen, Graphs on Surfaces, Johns Hopkins Studies in the Mathematical Sciences, Johns Hopkins University Press, Baltimore, MD, ISBN 0-8018-6689-8, 2001, xii+291 pp.

[10] C.St.J.A. Nash-Williams, Unexplored and semi-explored territories in graph theory, in: New Directions in Graph Theory, Academic Press, New York, 1973, pp. 169-176.

[11] R. Thomas, X. Yu, 4-Connected projective planar graphs are Hamiltonian, J. Combin. Theory Ser. B 62 (1994) 114-132.

[12] C. Thomassen, A theorem on paths in planar graphs, J. Graph Theory 7 (1983) 165-169.

[13] W.T. Tutte, A theorem on planar graphs, Trans. Amer. Math. Soc. 82 (1956) 99-116. 